\title{
Composición Factorial de la Escala de Locus de Control de Rotter en Universitarios Mexicanos
}

\author{
María A. Bibiano, Martha Ornelas, Juan F. Aguirre y Judith M. Rodríguez-Villalobos* \\ Universidad Autónoma de Chihuahua. Facultad de Ciencias de la Cultura Física. DES Salud CA 101 y \\ 121. Calle Escorza 900, CP 31000 Chihuahua, Chih.- México. (e-mail: mbibiano@uach.mx, \\ mornelas@uach.mx, jaguirre@uach.mx y jurodrig@uach.mx)
}

* Autor a quien debe ser dirigida la correspondencia

Recibido Abr. 14, 2016; Aceptado Jun. 1, 2016; Versión final Jul. 21, 2016, Publicado Dic. 2016

\section{Resumen}

Se analiza la composición factorial de la Escala de Locus de Control de Rotter en Universitarios Mexicanos y se compara con otros resultados presentados en la literatura. La muestra total fue de 1537 alumnos de las licenciaturas de Educación Física y Motricidad Humana que se ofrecen en la Universidad Autónoma de Chihuahua en México, con una edad media de 20.56 años. La estructura factorial del cuestionario se analizó a través de análisis factoriales confirmatorios. Los análisis, muestran que una estructura tetra factorial es adecuada. La estructura de cuatro factores (suerte, poder político, destino y relaciones interpersonales) ha mostrado adecuados indicadores de ajuste de validez. Además, los resultados de los análisis factoriales llevados a cabo con las submuestras, indican la existencia de fuertes evidencias de la estabilidad de la estructura factorial.

Palabras clave: estilos atributivos; estructura factorial; validación de constructo; ecuaciones estructurales

\section{Factor structure of the Rotter's Locus of Control Scale in Mexican university students}

\begin{abstract}
The factor structure of the Rotter's Locus of Control Scale in Mexican university students is analyzed. The sample consisted of 1537 participants; all of them students of the degree of Physical Education and Human Motricity offered at the Autonomous University of Chihuahua in Mexico, with a mean age of 20.56 years. Psychometric analysis showed that a tetra-factorial structure was adequate. The factorial structure of the questionnaire was analyzed with confirmatory factor analysis. The structure of the four factors (luck, political power, destiny and interpersonal relationships) showed adequate indicators of validity adjustment. In addition, factor analysis conducted with subsamples indicated the presence of strong evidence of factor structure stability.
\end{abstract}

Keywords: attributional style; factor structure; construct validation; structural equation 


\section{INTRODUCCIÓN}

El ser humano tiene una necesidad inherente de control. Las personas se esfuerzan por tener cierto grado de control sobre hechos que consideran significativos en sus vidas, logrando con esto alcanzar sus metas. La sensación de no tener control sobre un evento provoca a menudo un estado de inmovilización que incapacita a las personas para lograr las metas propuestas (Brenlla y Vázquez, 2010; Inozu et al., 2012; Oros, 2005); por ello cuando una persona siente que cambiar algún aspecto importante de su contexto está fuera de su alcance $o$ no puede prever un evento futuro, tiende a perder las ganas y la voluntad para modificar aquellos aspectos del entorno que son susceptibles de cambio; esta pérdida de ganas y de voluntad lleva a la persona a una actuación poco efectiva en los niveles cognitivo y conductual, así como al surgimiento de rasgos de indefensión, depresión y desesperanza (Karatas y Tagay, 2012).

Rotter (1966), en su teoría del aprendizaje social, describe cuatro tipos de variables que intervienen en la adquisición de habilidades y conocimiento: a) el potencial para llevar a cabo una conducta, b) las expectativas de reforzamiento, c) el valor del reforzamiento y d) la situación psicológica. Esta teoría sostiene que el potencial para que una persona lleve a cabo una conducta determinada en una situación específica depende de su expectativa sobre las consecuencias que tendrá la conducta y del valor que tienen esas consecuencias para la persona en ese determinado contexto (Cobb-Clark, 2015). Se afirma entonces que, la conducta de las personas podría predecirse desde la noción de sus expectativas sobre su propia conducta y los resultados a consecuencia de ella (Durán-Aponte y Pujol, 2013; Richardson et al., 2012); así pues, para la teoría del aprendizaje social, las expectativas tienen un rol importante y se distinguen los siguientes tipos: las expectativas generalizadas para el éxito, las expectativas generalizadas de confianza interpersonal y las expectativas generalizadas de control del refuerzo (Rotter, 1966).

El locus de control, ubicado en las expectativas generalizadas de control del refuerzo, permite clasificar a los sujetos en internos o externos (Rotter, 1966). Los sujetos con locus de control interno tendrían la expectativa de que los resultados que logran son contingentes u ocasionados por sus conductas o por sus características personales, lo que hace que se perciban como capaces de influir en su propio destino, de transformar una situación desfavorable, o de incrementar su probabilidad de éxito (Berglund et al., 2014; Sagone y De Caroli, 2014). La percepción de control sobre la situación aumenta la motivación para enfrentarla, de esta manera se espera que las personas con un locus de control interno se sientan más comprometidas, y actúen de forma más activa ante la situación. Mientras que las personas con un locus de control externo apreciarían que los resultados de sus conductas obedecen a factores ajenos a su control, como la suerte, el destino o la participación de otras personas, no reconociendo en ellas mismas la capacidad de afectar el curso de los eventos y de influir mediante sus acciones en el control de las contingencias de refuerzo que seguirán a su comportamiento (Durán-Aponte y Pujol, 2013; Rotter, 1966; Salvador y Mayoral, 2011).

Estar convencido de la posibilidad de afectar el funcionamiento del mundo a nuestro alrededor (locus de control interno) ha demostrado ser un elemento positivo en el rendimiento educativo (Sagone y De Caroli, 2014), el campo laboral (Malik et al., 2015) y la prevención de la salud (Cobb-Clark et al., 2014). De ahí que el locus de control interno, la autoeficacia y la resiliencia, entre otros, permiten afrontar los retos y alcanzar los fines deseados, además de favorecer la adaptación a las inevitables pérdidas que ocurren a lo largo de la vida (González-Arratia et al., 2012; Khoury y Günther, 2009).

Luego de su publicación en 1966 y pese a que ha sido duramente criticada por sus características psicométricas y por la forma, enunciado y contenido de sus ítems, la escala de Locus de Control InternoExterno (E I-E) de Rotter ha sido el instrumento de referencia para medir dicha dimensión de personalidad (Furnham y Steele, 1993). De acuerdo a Ferrando et al. (2011), esto puede deberse a la utilidad que parece tener en una amplia variedad de situaciones y contextos. Estos autores realizaron una evaluación del funcionamiento y propiedades de medida de la versión española de la $E$ I-E; utilizando un enfoque basado en la Teoría de Respuesta al Ítem y de la unidimensionalidad mediante análisis factorial semiconfirmatorio. Los pesos de los factores de primer orden resultaron todos por encima de 0.30 y una considerable proporción de la varianza de los factores de primer orden fue residual, Estos resultados mostraron un factor general bien definido e identificable aunque no se trate de un factor fuerte.

Por todo lo anteriormente expuesto el presente estudio instrumental (Montero y León, 2005) se ha dirigido a proporcionar apoyo empírico a la división factorial de la escala de Locus de control de Rotter; lo que se justifica por la importancia de comprobar la estructura factorial de un cuestionario y la equivalencia psicométrica del mismo en distintos grupos; ya que en el contexto de la comparación intergrupal, es indispensable plantearse la necesidad de llevar a cabo la adaptación de un instrumento de medida psicológica que cumpla con todos los criterios de equivalencia, pero sobre todo plantearse si la misma 
estructura factorial es aplicable a distintos grupos de sujetos 0 , de modo más genérico, a distintas poblaciones (Abalo et al., 2006).

\section{METODOLOGÍA}

Se detallan los participantes, luego se presenta el instrumento para la medición del locus de control, se explica el procedimiento y finalmente se discute sobre cómo se realiza el análisis de datos

\section{Participantes}

La muestra de 1537 participantes se obtuvo mediante un muestreo por conveniencia, tratando de abarcar la representatividad de las diferentes licenciaturas que se ofrecen en la Facultad de Ciencias de la Cultura Física (FCCF) de la Universidad Autónoma de Chihuahua. La edad de los participantes fluctuó entre los 18 y 26 años, con una media de 20.56 y una desviación estándar de 1.88 años. La muestra fue aleatoriamente dividida en dos partes utilizando el Statistical Package for the Social Sciences (SPSS) en su versión 18.0; con el fin de realizar estudios paralelos que permitieran corroborar y verificar los resultados obtenidos (validación cruzada). La submuestra 1 quedo constituida por 740 sujetos, 378 mujeres y 362 hombres. Las edades fluctúan entre los 18 y 26 años, con una media de 20.62 y una desviación estándar de 1.83 años. La submuestra 2 quedo compuesta por 797 sujetos, 442 mujeres y 355 hombres. Las edades fluctúan entre los 18 y 26 años, con una media de 20.51 y una desviación estándar de 1.93 años.

\section{Instrumento}

Para la medición del locus de control se utilizó la adaptación al castellano de Pérez (1984) de la Escala de Locus de Control de Rotter compuesta por 23 ítems.

\section{Procedimiento}

Se invitó a participar en el estudio a los alumnos de las licenciaturas que se ofrecen en la Facultad de Ciencias de la Cultura Física de la Universidad Autónoma de Chihuahua. Los que aceptaron participar firmaron la carta de aceptación correspondiente. Luego se aplicó el instrumento, antes descrito, por medio de una computadora personal (módulo administrador del instrumento del editor de escalas de ejecución típica), en una sesión de aproximadamente 30 minutos; en los laboratorios o centros de cómputo de la FCCF. Al inicio de cada sesión se hizo una pequeña introducción sobre la importancia de la investigación y de cómo acceder al instrumento. Se les solicitó la máxima sinceridad y se les garantizó la confidencialidad de los datos que se obtuvieran. Las instrucciones de cómo responder se encontraban en las primeras pantallas; antes del primer reactivo del instrumento. Al término de la sesión se les agradeció su participación. Una vez aplicado el instrumento se procedió a recopilar los resultados por medio del módulo generador de resultados del editor de escalas versión 2.0 (Blanco et al., 2013).

\section{Análisis de datos}

El primer paso del análisis de las propiedades psicométricas del cuestionario consistió en calcular la media, la desviaciones estándar, la asimetría y la curtosis de cada ítem. Para luego eliminar de la escala aquellos que obtienen una curtosis o asimetría extremas. Luego, para determinar el número mínimo de factores comunes capaces de reproducir, de un modo satisfactorio, las correlaciones observadas entre los ítems del instrumento, se realizó un análisis factorial exploratorio en la muestra total a partir del método de máxima verosimilitud, además de que para garantizar una adecuada representación de las variables (ítems), solo se conservaron aquéllos cuya carga factorial fuera superior a .40 en alguno de los factores retenidos después de una rotación varimax (Costello y Osborne, 2005).

Posteriormente, se sometieron a comparación dos modelos de medida: el Modelo 1 (M1), modelo de un factor acorde a la distribución original de los ítems dentro del cuestionario y el Modelo 2 (M2), que responde a un modelo de cuatro factores acorde a los resultados del análisis factorial exploratorio eliminando los ítems que no fueron suficientemente bien explicados. Para conducir los análisis factoriales confirmatorios se utilizó el software AMOS 21 (Arbuckle, 2012), las varianzas de los términos de error fueron especificados como parámetros libres, en cada variable latente (factor) se fijó uno de los coeficientes estructurales asociados a uno, para que su escala sea igual a la de una de las variables observables (ítems). El método de estimación empleado fue el de Máxima Verosimilitud; siguiendo la recomendación de Thompson (2004), en el sentido de que cuando se emplea análisis factorial confirmatorio se debe corroborar no sólo el ajuste de un modelo teórico sino que es recomendable comparar los índices de ajuste de varios modelos alternativos para seleccionar el mejor. 
Para evaluar el ajuste del modelo se emplearon el estadístico Chi-cuadrado, el índice de bondad de ajuste (GFI) y el error cuadrático medio de aproximación (RMSEA) como medidas absolutas de ajuste. El índice de bondad ajustado (AGFI), el Índice Tucker-Lewis (TLI) y el índice de bondad de ajuste comparativo (CFI) como medidas de ajuste incremental. La razón de Chi-cuadrado sobre los grados de libertad (CMIN/GL) y el Criterio de Información de Akaike (AIC) como medidas de ajuste de parsimonia (Byrne, 2010; Gelabert et al., 2011). Por último, siguiendo las recomendaciones de Abalo et al. (2006), se llevó a cabo un análisis de la invarianza factorial del cuestionario para las submuestras tomando como base el mejor modelo de medida obtenido en la etapa anterior. Por último se calculó la fiabilidad de cada una de las dimensiones, de los modelos de medida obtenidos en cada submuestra, a través del Coeficiente Alpha de Cronbach (Elosua y Zumbo, 2008; Nunnally y Bernstein, 1995) y del Coeficiente Omega (Revelle y Zinbarg, 2009; Sijtsma, 2009).

\section{RESULTADOS}

Los resultados se presentan para mayor claridad en cuatro sub secciones: i) análisis descriptivos; ii) análisis factorial exploratorio; iii) análisis factoriales confirmatorios; y iv) invarianza de la estructura factorial entre las sub-muestras.

\section{Análisis descriptivos}

En la Tabla 1 se resumen los resultados de los análisis descriptivos de cada uno de los 23 ítems del cuestionario en la muestra total. Las respuestas a todos los ítems reflejan unas puntuaciones medias que oscilan entre 0.08 y 0.79 , y la desviación estándar ofrece, en todos los casos, valores mayores a 0.25 (dentro de un rango de respuesta entre 0 y 1). La mayoría de los valores de asimetría y curtosis se encuentran dentro del rango \pm 3.0 ; por lo que se infiere que las variables se ajustan razonablemente a una distribución normal.

Tabla 1. Análisis descriptivos de los ítems del cuestionario "Escala de Locus de Control de Rotter". Muestra total.

\begin{tabular}{ccccc}
\hline Ítem & $\mathrm{M}$ & $\mathrm{DE}$ & $\mathrm{AS}$ & $\mathrm{CU}$ \\
\hline Ítem 1 & 0.08 & 0.27 & 3.18 & 8.14 \\
Ítem 2 & 0.67 & 0.47 & -0.74 & -1.45 \\
Ítem 3 & 0.70 & 0.46 & -0.85 & -1.27 \\
Ítem 4 & 0.79 & 0.41 & -1.39 & -0.07 \\
Ítem 5 & 0.23 & 0.42 & 1.30 & -0.30 \\
Ítem 6 & 0.69 & 0.46 & -0.82 & -1.33 \\
Ítem 7 & 0.56 & 0.50 & -0.26 & -1.93 \\
Ítem 8 & 0.30 & 0.46 & 0.89 & -1.21 \\
Ítem 9 & 0.20 & 0.40 & 1.49 & 0.23 \\
Ítem 10 & 0.69 & 0.46 & -0.83 & -1.31 \\
Ítem 11 & 0.31 & 0.46 & 0.84 & -1.29 \\
Ítem 12 & 0.11 & 0.31 & 2.53 & 4.40 \\
Ítem 13 & 0.08 & 0.27 & 3.07 & 7.41 \\
Ítem 14 & 0.51 & 0.50 & -0.02 & -2.00 \\
Ítem 15 & 0.42 & 0.49 & 0.32 & -1.90 \\
Ítem 16 & 0.54 & 0.50 & -0.15 & -1.98 \\
Ítem 17 & 0.54 & 0.50 & -0.15 & -1.98 \\
Ítem 18 & 0.53 & 0.50 & -0.14 & -1.98 \\
Ítem 19 & 0.14 & 0.35 & 2.04 & 2.16 \\
Ítem 20 & 0.42 & 0.49 & 0.32 & -1.90 \\
Ítem 21 & 0.64 & 0.48 & -0.60 & -1.65 \\
Ítem 22 & 0.13 & 0.33 & 2.26 & 3.12 \\
Ítem 23 & 0.43 & 0.50 & 0.28 & -1.92 \\
\hline
\end{tabular}

\section{Análisis factorial exploratorio}

La significación del Test de Bartlett $(843.499, \mathrm{p}<$.001) y la medida de adecuación muestral KMO (.637) mostraron una adecuada correlación entre los ítems y una buena adecuación muestral respectivamente, evidenciando la pertinencia de un análisis factorial. El análisis factorial exploratorio en la muestra total, 
después de una rotación varimax (Costello y Osborne, 2005), eliminando 11 de los 23 ítems analizados que no fueron suficientemente bien explicados, puso de manifiesto una estructura factorial de cuatro factores Suerte (ítems 8, 9, 12 y 22), Poder Político (ítems 10, 14 y 18), Destino (7 y 17) y Relaciones Interpersonales (ítems $6,16,21$ ). El conjunto de los factores retenidos explicaron el $40.25 \%$ de la varianza.

\section{Análisis factoriales confirmatorios}

Los resultados globales del análisis factorial confirmatorio en la submuestra 1 (GFI .934; RMSEA .042; CFI .552) y la submuestra 2 (GFI .931; RMSEA .046; CFI .540) para el modelo M1 que corresponde a la distribución original de los ítems dentro de la Escala de Locus de Control de Rotter, indican que el modelo de medición, en ambas submuestras se puede considerar como no aceptable (Tabla 2). En la Tabla 2 se tiene que ${ }^{*} \mathrm{p}<.05 ; \mathrm{GFI}=$ índice de bondad de ajuste; RMSEA = raíz del error medio; $\mathrm{AGFI}=$ índice corregido de la bondad de ajuste; TLI = índice de Tucker-Lewis; CFI = índice de ajuste comparativo; CMIN/DF = índice de ajuste chi cuadrado dividido por los grados de libertad; AIC = criterio de información de Akaike

Tabla 2 Índices absolutos, incrementales y de parsimonia para los modelos generados. Submuestras 1 y 2.

\begin{tabular}{|c|c|c|c|c|c|c|c|c|}
\hline \multirow[b]{2}{*}{ Modelo } & \multicolumn{3}{|c|}{ Índices absolutos } & \multicolumn{3}{|c|}{ Índices incrementales } & \multicolumn{2}{|c|}{ Índices de parsimonia } \\
\hline & $\chi^{2}$ & GFI & RMSEA & AGFI & TLI & $\mathrm{CFI}$ & CMIN/DF & AIC \\
\hline \multicolumn{9}{|c|}{ Primera solución factorial (submuestra 1) } \\
\hline M1 & $531.357^{*}$ & .934 & .042 & .921 & .507 & .552 & 2.310 & 623.357 \\
\hline M2 & $71.737^{\star}$ & .984 & .026 & .974 & .904 & .930 & 1.495 & 131.737 \\
\hline \multicolumn{9}{|c|}{ Segunda solución factorial (submuestra 2) } \\
\hline M1 & $611.409^{*}$ & .931 & .046 & .917 & .494 & .540 & 2.658 & 703.409 \\
\hline M2 & $69.798^{*}$ & .985 & .024 & .976 & .934 & .952 & 1.454 & 129.798 \\
\hline
\end{tabular}

El factor del modelo M1 explica aproximadamente el 10\% de la varianza en la primer y segunda submuestras. Por otro lado, ninguno de los 23 ítems, en ambas submuestras, saturan por encima de .40 en su dimensión prevista. Los resultados globales del análisis factorial confirmatorio en la primer (GFI .984; RMSEA .026; CFI .930) y segunda submuestra (GFI .985; RMSEA .024; CFI .952), del segundo modelo sometido a prueba (M2) que responde a un modelo de cuatro factores acorde a los resultados del análisis factorial exploratorio, indican que este modelo de medición es mejor que el modelo anterior y que su ajuste es aceptable (Tabla 2). Los cuatro factores de este modelo explican en conjunto, en ambas submuestras aproximadamente el $46 \%$ de la varianza. Por otro lado, de acuerdo a los resultados de la Tabla 3; solo tres ítems, en ambas submuestras, saturan por debajo de .40 en su dimensión prevista. Observándose además, intercorrelaciones de bajas a moderadas entre los factores evidenciando una adecuada validez discriminante entre ellos.

\section{Invarianza de la estructura factorial entre las submuestras}

Los índices de ajuste obtenidos (Tabla 4) permiten aceptar la equivalencia de los modelos de medida básicos entre las dos submuestras. Aunque el valor de Chi-cuadrado excede al exigido para aceptar la hipótesis de invarianza, los índices $\mathrm{GFI}=.985, \mathrm{CFI}=.942$, RMSEA=.018 y $\mathrm{AlC}=261.535$ contradicen esta conclusión lo que nos permite aceptar el modelo base de la invarianza (modelo sin restricciones).

Añadiendo al modelo base restricciones sobre las cargas factoriales caracterizamos la invarianza métrica. Los valores que se recogen en la tabla 4 permiten aceptar este nivel de invarianza. El índice de ajuste general (GFI .983) y el error cuadrático medio de aproximación (RMSEA .018) siguen aportando información convergente en esta dirección. Además, el criterio de información de Akaike (AIC 259.060) y el índice comparativo de Bentler (CFI .935) no sufren grandes variaciones respecto al modelo anterior. Haciendo uso del criterio para la evaluación de los modelos anidados propuesto por Cheung y Rensvold (2002), quiénes sugieren que si el cálculo de la diferencia de los CFI de ambos modelos anidados disminuye en .010 menos, se da por bueno el modelo restringido y por tanto el cumplimiento de la invarianza factorial; la diferencia entre CFIs obtenida permite aceptar el modelo de invarianza métrica. Podemos concluir hasta ahora que las cargas factoriales son equivalentes en las dos submuestras.

Una vez demostrada la invarianza métrica entre las submuestras, pasamos a evaluar la equivalencia entre interceptos (invarianza factorial fuerte). Los índices (Tabla 4) muestran un ajuste aceptable de este modelo, 
tanto evaluado de modo independiente como analizándolo respecto a su anidamiento con el modelo de invarianza métrica. La diferencia entre los índices comparativos de Bentler es .003; el índice de ajuste general es .983 y el error cuadrático medio de aproximación es .017. Aceptada la invarianza fuerte, los dos modelos evaluados son equivalentes respecto a los coeficientes factoriales y a los interceptos. Los factores obtenidos en los análisis factoriales confirmatorios alcanzan valores de consistencia interna por debajo de .75 en ambas submuestras evidenciando una baja consistencia interna (Tabla 5).

Tabla 3 Soluciones estandarizadas análisis factorial confirmatorio para el Modelo M2. Submuestra 1 y 2 Nota: F1 = Suerte, F2 = Poder Político, F3 = Destino, F4 = Relaciones Interpersonales

\begin{tabular}{|c|c|c|c|c|c|c|c|c|}
\hline \multirow[b]{2}{*}{ Item } & \multicolumn{4}{|c|}{ Submuestra 1} & \multicolumn{4}{|c|}{ Submuestra 2} \\
\hline & $\mathrm{F} 1$ & $\mathrm{~F} 2$ & F3 & $\mathrm{F} 4$ & $\mathrm{~F} 1$ & $\mathrm{~F} 2$ & $\mathrm{~F} 3$ & $\mathrm{~F} 4$ \\
\hline \multicolumn{9}{|c|}{ Pesos Factoriales } \\
\hline $\begin{array}{l}8 \text { Muchas veces las preguntas de los exámenes tienden a estar } \\
\text { tan poco relacionadas con el trabajo en el curso, que estudiar } \\
\text { resulta realmente inútil. }\end{array}$ & .43 & & & & .45 & & & \\
\hline $\begin{array}{l}9 \text { Conseguir un buen trabajo depende, principalmente, de estar } \\
\text { en el lugar correcto, en el momento oportuno. }\end{array}$ & .39 & & & & .59 & & & \\
\hline $\begin{array}{l}12 \text { Muchas veces podríamos decidir qué hacer simplemente } \\
\text { tirando una moneda. }\end{array}$ & .44 & & & & .38 & & & \\
\hline $\begin{array}{l}22 \text { A veces siento que no tengo suficiente control sobre el rumbo } \\
\text { que está tomado mi vida. }\end{array}$ & .48 & & & & .40 & & & \\
\hline $\begin{array}{l}10 \text { Este mundo está manejado por unas pocas personas que se } \\
\text { encuentran en el poder, y el hombre común no puede hacer } \\
\text { mucho respecto de ello. }\end{array}$ & \multicolumn{4}{|c|}{.45} & \multicolumn{4}{|c|}{.60} \\
\hline $\begin{array}{l}14 \text { En relación a los sucesos mundiales, la mayoría de nosotros } \\
\text { somos las víctimas de fuerzas que no podemos ni entender ni } \\
\text { controlar. }\end{array}$ & \multicolumn{4}{|c|}{.43} & \multicolumn{4}{|c|}{.36} \\
\hline $\begin{array}{l}18 \text { Es difícil para la gente tener demasiado control sobre lo que } \\
\text { hacen los políticos. }\end{array}$ & \multicolumn{4}{|c|}{.51} & \multicolumn{4}{|c|}{.47} \\
\hline 7 A menudo he notado que lo que tiene que suceder, sucederá. & \multicolumn{4}{|c|}{.49} & \multicolumn{4}{|c|}{.65} \\
\hline $\begin{array}{l}17 \text { A la larga, lo malo que nos sucede se compensa con lo bueno } \\
\text { que nos pasa. }\end{array}$ & \multicolumn{4}{|c|}{.34} & \multicolumn{4}{|c|}{.37} \\
\hline $\begin{array}{l}6 \text { No importa lo que hagas, siempre habrá alguien a quien no le } \\
\text { caes bien. }\end{array}$ & & & & .53 & & & & .62 \\
\hline 16 Es difícil saber si, realmente, le agradas o no a una persona. & & & & .24 & & & & .32 \\
\hline $\begin{array}{l}21 \text { No tiene mucho sentido tratar de agradar a la gente; si les } \\
\text { gustas, les gustas. }\end{array}$ & & & & .29 & & & & .24 \\
\hline \multicolumn{9}{|c|}{ Correlaciones Factoriales } \\
\hline $\mathrm{F} 1$ & - & & & & - & & & \\
\hline $\mathrm{F} 2$ & .28 & - & & & .36 & - & & \\
\hline F3 & .00 & .26 & - & & .00 & .12 & - & \\
\hline $\mathrm{F} 4$ & .00 & .24 & .46 & - & .00 & .11 & .26 & - \\
\hline
\end{tabular}

Tabla 4 Índices de bondad de ajuste de cada uno de los modelos puestos a prueba en la invarianza factorial. * $\mathrm{p}<.05 ; \mathrm{GFI}=$ índice de bondad de ajuste; $\mathrm{NFI}=$ índice de ajuste normado; $\mathrm{CFI}=$ índice de ajuste comparativo; RMSEA = raíz del error medio; AIC = criterio de Información de Akaike

\begin{tabular}{lccccccc}
\hline \multicolumn{1}{c}{ Modelo } & \multicolumn{7}{c}{ Índice de Ajuste } \\
\cline { 2 - 8 } & $\chi^{2}$ & gl & GFI & NFI & CFI & RMSEA & AIC \\
\hline Modelo sin restricciones & $141.535^{*}$ & 96 & .985 & .846 & .942 & .018 & 261.535 \\
Invarianza métrica & $155.060^{*}$ & 104 & .983 & .832 & .935 & .018 & 259.060 \\
Invarianza factorial fuerte & $161.105^{*}$ & 112 & .983 & .825 & .938 & .017 & 249.105 \\
\hline
\end{tabular}


Tabla 5. Coeficiente omega y alfa para los factores obtenidos en los análisis factoriales confirmatorios submuestras 1 y 2

\begin{tabular}{lcccc}
\hline & \multicolumn{2}{c}{ Submuestra 1 } & \multicolumn{2}{c}{ Submuestra 2 } \\
\cline { 2 - 5 } Factor & $\Omega$ & $\alpha$ & $\Omega$ & $\alpha$ \\
\hline Suerte & .483 & .470 & .513 & .515 \\
Poder Político & .451 & .452 & .472 & .459 \\
Destino & .295 & .284 & .419 & .386 \\
Relaciones Interpersonales & .304 & .308 & .362 & .334 \\
\hline
\end{tabular}

\section{DISCUSIÓN}

El objetivo principal del estudio fue indagar si se replican o no los resultados psicométricos propuestos por Ferrando et al. (2011) para la escala de locus de control de Rotter a través de una muestra de universitarios utilizando el análisis factorial confirmatorio (AFC). Los análisis factoriales confirmatorios realizados en cada submuestra por separado apoyan una estructura factorial de cuatro factores: Suerte, Poder Político, Destino y Relaciones Interpersonales, donde los factores así obtenidos presentan en general saturaciones factoriales estandarizadas moderadas, sugiriendo además la existencia de fuertes evidencias de la validación cruzada de la medida y por tanto de la estabilidad de la estructura hasta que no se demuestre lo contrario. No obstante, la consistencia interna de cada uno de ellos es baja, probablemente debido al número reducido de ítems en cada uno de ellos.

En síntesis, el análisis de las propiedades psicométricas de la escala de locus de control de Rotter, ha mostrado que una estructura tetrafactorial es viable y adecuada de acuerdo a los requisitos psicométricos establecidos. La estructura de cuatro factores, salvo por la baja consistencia interna de sus factores y las saturaciones factoriales estandarizadas moderadas ha mostrado adecuados indicadores de ajuste y de validez.

Por otro lado, el modelo obtenido no coincide con el planteado por Ferrando et al. (2011), ya que para lograr un mejor ajuste hubo que eliminar 11 de los 23 ítems analizados y cambiar la saturación original de algunos ítems; esto último tomando como base a los índices de modificación y a su justificación teórica. Las discrepancias observadas entre el modelo propuesto por Ferrando et al. (2011) y el aquí presentado pueden atribuirse, entre otros, a factores tales como la escala de medición empleada ( 0 y 1 ); la diversidad de situaciones referidas en los ítems y a diferencias sociales o culturales de los participantes; como el de ser estudiantes universitarios del área de la actividad física. En todo caso, la validación de un cuestionario es un proceso lento y continuo, por lo que, futuras investigaciones deberán contrastar estos hallazgos en muestras más amplias (Holgado et al., 2009).

Sin embargo, cabe señalar que el alcance de estos resultados es limitado y por ello es necesario que en investigaciones futuras se confirme la estructura obtenida, lo cual permitirá contar con evidencia más robusta respecto a la estructura factorial de la escala. Específicamente, debe demostrarse si la invarianza de la estructura de la escala se cumple por género, edad, entre alumnos de distintas licenciaturas, entre otras; de tal manera que, se considera que más estudios son necesarios con el fin de corroborar o refutar los datos obtenidos en las investigaciones realizadas hasta el momento. Además de retomar la validación cuestionario incrementando el número de ítems en cada uno de sus factores con el fin de mejorar su confiabilidad y validez. Asimismo, es indispensable comprobar si la escala resulta útil para estudiar la relación entre locus de control y variables como el rendimiento académico, la resiliencia y el bienestar psicológico percibidos en universitarios.

\section{CONCLUSIONES}

De acuerdo a lo presentado y discutido en este trabajo, se pueden obtener las siguientes conclusiones principales:

1) El Análisis Factorial Confirmatorio, en ambas submuestras, indicó que el ajuste de los datos al modelo teórico de 12 reactivos agrupados en cuatro factores es aceptable. No obstante, la consistencia interna de cada uno de ellos es baja

2) Los resultados del análisis de la invarianza factorial entre las submuestras; indican una alta congruencia entre pares de factores. 
3) Cuando menos tres limitaciones están presentes en este trabajo. La primera es que los participantes son solo estudiantes del área de la actividad física, lo que supone una amenaza para la posibilidad de generalizar estos resultados. Ampliar la muestra (agregando por ejemplo adultos jóvenes o adolescentes que no son estudiantes) es un área de trabajo de cara al futuro. La segunda limitación proviene del propio instrumento de evaluación, que se basa en el autoinforme y que puede contener los sesgos que se derivan de la deseabilidad social. La tercera se refiere a su escala de medición dicotómica, lo reduce la posibilidad obtener diferencias (varianza).

\section{REFERENCIAS}

Abalo J., Lévy J., Rial A. y Varela J., Invarianza factorial con muestras múltiples, En Modelización con Estructuras de Covarianzas en Ciencias Sociales por J. Lévy, pp 259-278 Netbiblo, Madrid, (2006)

Arbuckle J. R., AMOS users guide version 21.0, Marketing Department, SPSS Incorporated, Chicago, IL, (2012)

Berglund E., Lytsy P. y Westerling R., The influence of locus of control on self-rated health in context of chronic disease: a structural equation modeling approach in a cross sectional study, BMC Public Health, 14(492), (2014)

Blanco H., Ornelas M., Tristán J. L., Cocca A., Mayorga-Vega D., López-Walle J., et al., Editor for creating and applying computerise surveys, doi: http://dx.doi.org/10.1016/j.sbspro.2013.12.105, Procedia Social and Behavioral Sciences, 106, 935-940, (2013)

Brenlla M. y Vázquez N. Análisis psicométrico de la adaptación argentina de la escala de locus de control de Rotter. Documento de trabajo. Santa María de los Bs As: Observatorio de la deuda social argentina; (2010)

Byrne B. M., Structural Equation Modeling With AMOS: Basic Concepts, Applications, and Programming, Routledge, New York, NY, (2010)

Cobb-Clark D., Locus of control and the labor market, doi: 10.1186/s40172-014-0017-x, IZA Journal of Labor Economics, 4(1), 1-19, (2015)

Cobb-Clark D., Kassenboehmer S. y Schurerb S., Healthy habits: The connection between diet, exercise, and locus of control, Journal of Economic Behavior \& Organization, 98 1- 28, (2014)

Costello A. B. y Osborne J. W., Best practices in exploratory factor analysis: four recommendations for getting the most from your analysis, Practical Assessment, Research and Evaluation, 10(7), 1-9, (2005)

Cheung G. W. y Rensvold R. B., Evaluating goodness-of-fit indexes for testing measurement invariance, doi: 10.1207/s15328007SEM0902_5, Structural Equation Modeling, 9(2), 233-255, (2002)

Durán-Aponte E. y Pujol L., Escala atribucional de motivación de logro general (eaml-g): Adaptación y análisis de sus propiedades psicométricas, Estudios Pedagógicos, XXXIX(1), 83-97, (2013)

Elosua P. y Zumbo B. D., Coeficientes de fiabilidad para escalas de respuesta categórica ordenadas, Psicothema, 20(4), 896-901, (2008)

Ferrando P. J., Demestre J., Anguiano-Carrasco C. y Chico E., Evaluación TRI de la escala I-E de Rotter: un nuevo enfoque $y$ algunas consideraciones, Psicothema, 23(2), 282-288, (2011)

Furnham, A., y Steele, H., Measuring locus of control: A critique of general, children's health- and workrelated locus of control, British Journal of Psychology, 84(4), 443-479, (1993)

Gelabert E., García-Esteve L., Martín-Santos R., Gutiérrez F., Torres A. y Subirà S., Psychometric properties of the Spanish version of the Frost Multidimensional Perfectionism Scale in women, Psicothema, 23(1), 133139, (2011)

González-Arratia N., Valdez J., Van Barneveld H. y González S., Resiliencia y factores protectores en menores infractores y en situación de calle.pdf, Psicología y Salud, 22(1), 49-62, (2012)

Holgado F. P., Soriano J. A. y Navas L., El cuestionario de autoconcepto físico (CAF): análisis factorial confirmatorio y predictivo sobre el rendimiento académico global y específico del área de educación física, Acción Psicológica, 6(2), 93-102, (2009) 
Inozu M., Yorulmaz O. r. y Terzi S., Locus of control in obsessive-compulsive (OC) and depression symptoms: The moderating effect of externality and obsessive-related control beliefs in OC symptoms, doi: 10.1017/bec.2012.14, Behavior Change, 29, 148-163, (2012)

Karatas Z. y Tagay O., Self esteem, locus of control and multidimensional perfectionism as the predictors of subjetive well being, International Education Studies, 5(6), 131 - 139, (2012)

Khoury H. T. T. y Günther I. A., Processos de Auto-Regulação no Curso de Vida: Controle Primário e Controle Secundário, Psicologia: Reflexão e Crítica, 22(1), 144-152, (2009)

Malik M. A. R., Butt A. N. y Choi J. N., Rewards and employee creative performance: Moderating effects of creative self-efficacy, reward importance, and locus of control, Journal of Organizational Behavior, 59-74, (2015)

Montero I. y León O., Sistema de clasificación del método en los informes de investigación en Psicología, International Journal of Clinical and Health Psychology, 5, 115-127, (2005)

Nunnally J. C. y Bernstein I. H., Teoría Psicométrica, McGraw-Hill, México, (1995)

Oros L. B., Locus de control: Evolución de su concepto y operacionalización, Revista de Psicología de la Universidad de Chile, XIV(1), 00-00, (2005)

Pérez A. M., Dimensionalidad del constructo "Locus of Control", Revista de psicología general y aplicada, 39(3), 471-488, (1984)

Revelle W. y Zinbarg R. E., Coefficients alpha, beta, omega and the glb: comments on Sijtsma, doi: 10.1007/s11336-008-9102-z, Psychometrika, 74(1), 145-154, (2009)

Richardson M., Abraham C. y Bond R., Psychological correlates of university students' academic performance: A systematic review and meta-analysis, doi: 10.1037/a0026838, American Psychological Association, 138(2), 353-387, (2012)

Rotter J. B., Generalized expectancies for internal versus external control of reinforcement Psychological Monographs: General and Applied, 80(1), (1966)

Sagone E. y De Caroli M. E., Locus of control and academic self-efficacy in university students: the effects of Self-concepts, Procedia - Social and Behavioral Sciences, 114, 222 - 228, (2014)

Salvador C. y Mayoral L., Influencia de los estilos de atribución en la inteligencia emocional de los estudiantes argentinos, Revista Electrónica Interuniversitaria de Formación del Profesorado, 14(1), 243-251, (2011)

Sijtsma K., On the use, the misuse, and the very limited usefulness of Cronbach's alpha, doi: 10.1007/s11336-008-9101-0, Psychometrika, 74(1), 107-120, (2009)

Thompson B., Exploratory and Confirmatory Factor Analysis. Understanding concepts and applications. , American Psychological Association, Washington, D C, (2004) 
